\title{
Cuidados paliativos na emergência: revisão integrativa
}

Maria Olívia Sobral Fraga de Medeiros ${ }^{1}$, Mariana do Valle Meira ${ }^{1}$, Jacilene Santiago do Nascimento Trindade dos Santos ${ }^{1}$, Larissa Chaves Pedreira ${ }^{1}$, Anelise Coelho da Fonseca ${ }^{2}$, Rudval Souza da Silva ${ }^{3}$

1. Universidade Federal da Bahia, Salvador/BA, Brasil. 2. Hospital Adventista Silvestre, Rio de Janeiro/RJ, Brasil.

3. Universidade do Estado da Bahia, Senhor do Bonfim/BA, Brasil.

\section{Resumo}

O objetivo do artigo é conhecer a abordagem de equipes de emergência à assistência de pacientes com doença crônica avançada, na perspectiva dos cuidados paliativos. O texto traz resultados de revisão integrativa que buscou artigos em cinco bases de dados, utilizando os descritores "serviços médicos de emergência", "equipe de assistência ao paciente", "atitude do pessoal de saúde" e "cuidados paliativos". Inicialmente, foram identificadas 12.290 publicações, reduzidas, após análise, a uma amostra final de 26 artigos. Entre as principais medidas mencionadas na literatura para levar os cuidados paliativos à emergência, estão: plano de cuidados individualizado e flexível; gestão de redes; acesso à equipe de cuidados paliativos; comunicação empática; identificação dos pacientes elegíveis; e controle de sintomas. Conclui-se que as equipes de emergência precisam reconhecer a importância dos cuidados paliativos nesse serviço, redirecionando o cuidado concentrado em "salvar vidas" para um cuidado que preserve a dignidade humana.

Palavras-chaves: Equipe de assistência ao paciente. Serviço hospitalar de emergência. Cuidados paliativos.

\section{Resumen}

\section{Cuidados paliativos en servicios de emergencia: revisión integradora}

Este artículo tuvo como objetivo comprender el enfoque del equipo de emergencia a los pacientes con enfermedad crónica avanzada desde una perspectiva paliativa. Se realizó una revisión integradora, buscando artículos en portugués, inglés y español en las bases de datos MEDLINE, LILACS, SciELO, IBECS y CINAHL, utilizando los descriptores "servicios médicos de emergencia", "equipo de asistencia al paciente", "actitud del personal de salud" y "cuidados paliativos", con 12.290 publicaciones identificadas inicialmente, que tras su análisis dieron como resultado una muestra final de 26 artículos. Entre los principales aspectos destacan: plan de cuidados individualizado y flexible; gestión de redes; acceso al equipo de cuidados paliativos; comunicación empática; identificación de pacientes elegibles; y control de síntomas. Se concluye que el equipo de emergencias necesita reconocer la importancia de los cuidados paliativos en este servicio y reorientar los cuidados enfocados a "salvar vidas" hacia cuidados que "preserven la dignidad humana".

Palabras clave: Grupo de atención al paciente. Servicio de urgencia en hospital. Cuidados paliativos.

\section{Abstract}

\section{Palliative care in emergency services: an integrative review}

This article seeks to understand the approach of emergency teams to patients with an advanced chronic condition from a palliative care perspective. This integrative review searched for articles in five databases using the descriptors "emergency medical services," "patient assistance team," "attitudes of health personnel" and "palliative care". At first, 12,290 publications were identified, which were then reduced to 26 articles for the final sample. Among the main measures found in the literature to use palliative care in emergency services, the following stand out: individualized and flexible care plan; network management; access to the palliative care team; empathic communication; identification eligible patients; and control of symptoms. We thus conclude that emergency teams must recognize the importance of palliative care and redirect the care focused in "saving lives" towards a care that preserves human dignity. Keywords: Patient care team. Emergency service, hospital. Palliative care. 
Com os avanços na área da saúde, a redução da mortalidade e a ampliação do acesso aos serviços sanitários, as pessoas têm vivido mais em todo mundo. Todavia, tem se discutido bastante a questão da qualidade de vida, pois, junto com a expectativa de vida, também tem aumentado a prevalência de doenças crônico-degenerativas, como câncer, Alzheimer e esclerose múltipla ${ }^{1}$.

Diante desse cenário de adoecimento crônico, observa-se fragilidade da atenção primária, o que tem levado usuários a recorrer às emergências como meio mais fácil de acesso, disponível 24 horas por dia, sete dias por semana ${ }^{1}$. Nesses serviços é comum o atendimento a pacientes com dor, dispneia e vômito que, sem atendimento ambulatorial ou domiciliar eficaz, veem na emergência a única e imediata opção ${ }^{1-2}$. Esses pacientes esperam uma assistência resolutiva, compassiva e individualizada. No entanto, estudos ${ }^{1-3}$ apontam certo distanciamento, quando se trata de pacientes em cuidados de fim de vida, por parte da equipe profissional.

Os pacientes assinalam que a busca pela emergência se dá principalmente pela falta de disponibilidade de uma equipe de cuidados paliativos. Entretanto, a equipe da emergência reconhece que não pode aplicar no atendimento os mesmos critérios usados em nível ambulatorial. $O$ argumento é que a dinâmica acelerada do serviço não permite que os profissionais dediquem um tempo maior para estar com o paciente e família e desenvolver uma interação mais próxima ${ }^{3}$.

Entretanto, embora não seja o local ideal para iniciar a assistência paliativa, a emergência poderia integrar o manejo de sintomas em crises agudas, desconstruindo uma cultura de atendimento apenas para casos agudos, de modo a dar espaço a um cuidado centrado no paciente, e não exclusivamente na doença. Isso já ocorre em alguns países desenvolvidos, como os Estados Unidos, onde foi criado o projeto Melhorando os Cuidados Paliativos nas Emergências Médicas (Improving Palliative Care in Emergency Medicine), um movimento embrionário de educação para equipes de emergência que define objetivos para avaliar o paciente, incluindo cuidados de fim de vida e manejo de sintomas ${ }^{1}$.

Considerando todo esse contexto, o presente estudo busca conhecer, por meio de uma revisão de literatura e desde a perspectiva dos cuidados paliativos, a abordagem de equipes de emergência na assistência a pacientes com doença crônica avançada.

\section{Método}

Este artigo traz resultados de revisão integrativa desenvolvida em seis etapas: definição do objeto e questão norteadora, busca na literatura, categorização, avaliação, interpretação e síntese do conhecimento ${ }^{4}$. Para elaborar a questão de pesquisa, utilizou-se a estratégia PICO: pacientes em paliação (patient), abordagem da equipe emergencista (intervention) e atendimento na emergência (outcomes). O terceiro elemento (comparison) não foi utilizado.

Foram incluídos artigos publicados entre junho de 2013 e junho de 2018, a partir de seleção por conveniência. A busca ocorreu entre julho e agosto de 2019, nas bases de dados Medical Literature Analysis and Retrieval System Online (Medline/PubMed), Literatura Latino-Americana e do Caribe em Ciências da Saúde (Lilacs), Scientific Electronic Library Online (SciELO), Índice Bibliográfico Español en Ciencias de la Salud (Ibecs) e Cumulative Index to Nursing and Allied Health Literature (Cinahl). Utilizaram-se os seguintes descritores, em português, inglês e espanhol, extraídos dos Descritores em Ciências da Saúde (DeCS) e Medical Subject Headings (MeSH): "emergency medical services", "patient care team", "attitude of health personnel" e "palliative care".

Foram incluídos textos em inglês, português ou espanhol que tratassem dos cuidados paliativos na emergência. Foram excluídos ensaios, artigos duplicados, artigos de revisão (integrativa ou sistemática), capítulos de livros, editoriais, teses e dissertações. A busca nas bases de dados resultou em 12.290 publicações, que compuseram o cenário global (Figura 1). Após aplicação dos critérios de inclusão e de exclusão, bem como de análise criteriosa com base no objetivo do estudo, realizada por dois pesquisadores independentes, chegou-se a uma amostra de 26 artigos, analisados na íntegra (Quadro 1).

Os princípios éticos foram observados, com a devida citação aos autores incluídos. Os resultados foram analisados de forma descritiva, a partir de síntese da abordagem de equipes de emergência à assistência a pacientes em cuidados paliativos e de comparações entre as pesquisas incluídas. 
Figura 1. Fluxograma do processo de seleção dos estudos

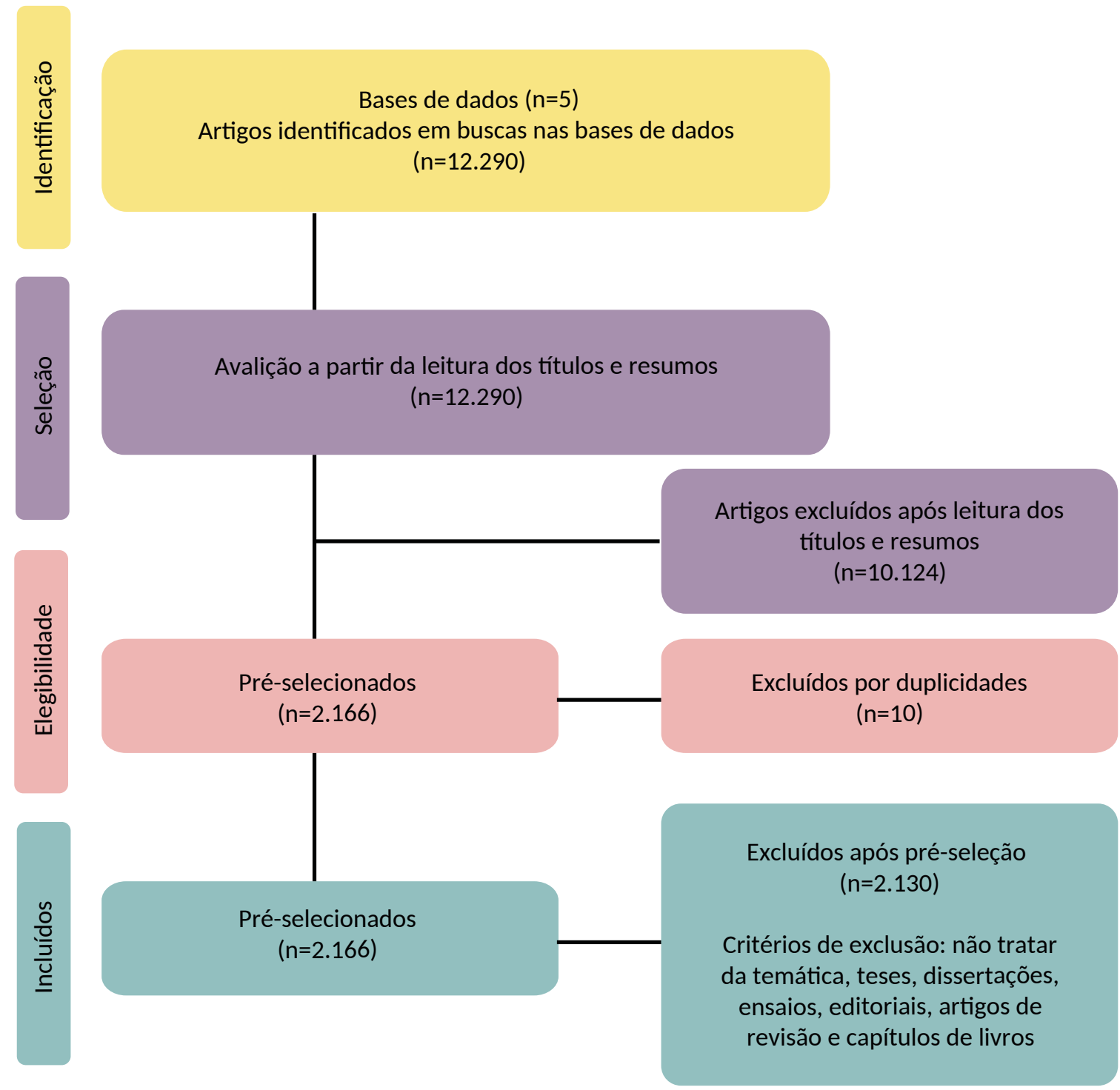

\section{Resultados}

As publicações incluídas na amostra têm diferentes abordagens, cobrindo tanto a perspectiva de pacientes e familiares como das equipes de saúde e administradores dos serviços. A maioria dos estudos foi publicada em 2014 $(26,9 \%, n=7)$. Os demais anos, 2013, 2015, 2016, 2017 e 2018, contaram com 5, 3, 5, 4 e 2 artigos, respectivamente. A abordagem mais utilizada foi a qualitativa, presente em 20 dos artigos. Os outros seis eram estudos quantitativos. Os países com maior número de publicações foram Estados Unidos $(38,4 \%, n=10)$ e Austrália $(30,8 \%, n=8)$. Quatro estudos eram do Reino Unido $(15,4 \%)$, e os quatro restantes foram realizados um em cada país: Espanha, França, Tailândia e Turquia. Predominaram publicações na língua inglesa, com apenas um estudo publicado em espanhol. A Tabela 1 apresenta as principais informações das publicações. 
Tabela 1. Principais estudos que compuseram a revisão integrativa

Base, periódico, ano, país Objetivo Abordagem por parte da equipe

Medline, J Palliat Med, 2013, EUA $^{3}$

Descobrir barreiras percebidas por médicos ao prestar cuidados paliativos no setor de emergência.

Descrever a abordagem e o papel da

Medline, West J Emerg

Med, 2013, EUA ${ }^{5}$ equipe de emergência quanto aos cuidados paliativos e de fim de vida.
- Identificação dos pacientes elegíveis.

- Acesso à equipe de cuidados paliativos.

- Identificação dos pacientes elegíveis.

- Acesso à equipe de cuidados paliativos.

- Gestão em rede de cuidados.

- Atuação em equipe interdisciplinar.

- Profissional da equipe de emergência como referência em cuidados paliativos.

- Plano de cuidados individualizado e flexível.

- Diferenciação entre cuidados paliativos e

cuidados de fim da vida.

Avaliar se interconsultas com equipes de cuidados paliativos iniciadas na

Cinahl, J Palliat Med, 2013, EUA $^{6}$ emergência diminuem o tempo de internação, em comparação com interconsultas iniciadas após internação hospitalar.

- Identificação de pacientes elegíveis.

- Acesso à equipe de cuidados paliativos.

- Gestão em rede de cuidados.

- Atuação em equipe interdisciplinar.

- Gestão em rede de cuidados.

- Plano de cuidados individualizado e flexível.

- Apoio à família. na emergência.

Cinahl, Am J Hosp Palliat Care, 2013, Irlanda ${ }^{8}$

Identificar pacientes em cuidados paliativos atendidos na emergência.

Avaliar a eficácia de estratégia de intervenção multimodal para melhorar os cuidados em fim de vida na emergência.

- Identificação dos pacientes elegíveis.

- Acesso à equipe de cuidados paliativos.

- Comunicação empática.

- Comunicação empática.

- Controle dos sintomas.

- Diferenciação entre cuidados paliativos e cuidados de fim da vida.

- Minimização de tratamentos fúteis.

- Adoção das diretivas antecipadas de vontade.

- Plano de cuidados individualizado e flexível.

Medline, Asian Pac

J Cancer Prev, 2014,

Turquia ${ }^{10}$

Identificar características de pacientes com diagnóstico oncológico internados no departamento de emergência.

- Controle dos sintomas.

- Gestão em rede de cuidados.

- Integração com a atenção domiciliar.

Explorar visões e experiências

Medline, Support Care Cancer, 2014, Austrália ${ }^{11}$

de interação interdisciplinar de profissionais de saúde que cuidam de pacientes com câncer avançado atendidos na emergência.

- Comunicação empática.

- Plano de cuidados individualizado e flexível.

- Gestão em rede de cuidados.

- Integração com a atenção domiciliar.

- Atuação em equipe interdisciplinar.

Medline, Emerg Med

Australas, 2014

Austrália ${ }^{12}$

Investigar perspectivas e necessidades de uma equipe de emergência em relação a cuidados paliativos.

- Controle dos sintomas.

- Comunicação empática.

- Plano de cuidados individualizado e flexível.

Avaliar barreiras e facilidades em relação a cuidados de fim de vida para pacientes com câncer atendidos na emergência.
- Plano de cuidados individualizado e flexível.

- Minimização de tratamentos fúteis.

- Adoção das diretivas antecipadas de vontade. 
Tabela 1. Continuação

Base, periódico, ano, país

Objetivo

Abordagem por parte da equipe

Medline, J Palliat Med, 2014, EUA $^{14}$

Identificar a taxa de interconsultas com a equipe de cuidados paliativos no departamento de emergência para pacientes com demência avançada.

- Identificação dos pacientes elegíveis.

- Acesso à equipe de cuidados paliativos.

Avaliar o encaminhamento precoce da emergência para a unidade de cuidados paliativos de pacientes

- Identificação dos pacientes elegíveis.

Medline, Acad Emerg

Med, 2014, EUA ${ }^{15}$ com câncer avançado.

- Acesso à equipe de cuidados paliativos.

Explorar a compreensão dos

Medline, Palliat Med 2015, Austrália ${ }^{16}$

Emerg Med, 2015, Austrália ${ }^{17}$

Medline, Emerg Med

Australas, 2015,

Austrália ${ }^{18}$

Medline, Ann Emerg Med, 2016, França ${ }^{19}$ cuidados paliativos por profissionais de saúde que cuidam de pacientes com câncer avançado num departamento de emergência.

Discutir atitudes de médicos de emergência quanto ao cuidado de pacientes com câncer avançado, pensando como suas atitudes afetam o acesso ao serviço de cuidados paliativos.

Investigar experiências e atitudes de equipe de emergência de um hospital público na abordagem dos cuidados paliativos.

Explorar percepções e atitudes dos médicos na tomada de decisão sobre a transferência de pacientes idosos em estado crítico do departamento de emergência para a UTI.

Descrever o significado de experiências

Medline, Int Nurs Rev, 2016, Tailândia ${ }^{20}$ vivenciadas por enfermeiros ao cuidar de pacientes críticos no departamento de emergência.

Descrever experiências de médicos

Medline, J Emerg Nurs, 2016, Espanha ${ }^{21}$ e enfermeiros em relação à perda da dignidade de uma pessoa em cuidados de fim de vida atendida num departamento de emergência.

Medline, J Emerg Nurs 2016, EUA ${ }^{22}$
Implementar um modelo de melhores estão morrendo. práticas para atender pacientes que
- Controle dos sintomas.

- Identificação dos pacientes elegíveis.

- Comunicação empática.

- Discussão dos dilemas éticos.

- Identificação dos pacientes elegíveis.

- Acesso à equipe de cuidados paliativos.

- Gestão em rede de cuidados.

- Controle dos sintomas.

- Apoio à família.

- Comunicação empática.

- Adoção das diretivas antecipadas de vontade.

- Identificação dos pacientes elegíveis.

- Comunicação empática.

- Adoção das diretivas antecipadas de vontade.

- Discussão dos dilemas éticos.

- Plano de cuidados individualizado e flexível.

- Minimização de tratamentos fúteis.

- Comunicação empática.

- Apoio à família.

- Apoio no processo de luto da família.

- Cuidados psicológicos e espirituais.

- Controle dos sintomas.

- Gestão em rede de cuidados.

- Plano de cuidados individualizado e flexível.

- Minimização de tratamentos fúteis.

- Adoção das diretivas antecipadas de vontade.

- Apoio à família.

- Apoio à equipe.

- Preservação da dignidade do paciente.

- Identificação dos pacientes elegíveis.

- Acesso à equipe de cuidados paliativos.

- Presença do enfermeiro especialista.

- Discussão ampliada entre a equipe interdisciplinar.

continua... 
Tabela 1. Continuação

\begin{tabular}{|c|c|c|}
\hline Base, periódico, ano, país & Objetivo & Abordagem por parte da equipe \\
\hline $\begin{array}{l}\text { Medline, BMJ Open, 2016, } \\
\text { Inglaterra }^{23}\end{array}$ & $\begin{array}{l}\text { Compreender o processo de tomada } \\
\text { de decisão de pessoas com câncer } \\
\text { avançado e seus cuidadores ao buscar } \\
\text { atendimento emergencial. }\end{array}$ & $\begin{array}{l}\text { - Atuação em equipe interdisciplinar. } \\
\text { - Controle dos sintomas. }\end{array}$ \\
\hline $\begin{array}{l}\text { Cinahl, J Pain Symptom } \\
\text { Manage, } 2017, \\
\text { Inglaterra }^{24}\end{array}$ & $\begin{array}{l}\text { Explorar atitudes de médicos em } \\
\text { relação à intubação de pacientes } \\
\text { com câncer em estado terminal. }\end{array}$ & $\begin{array}{l}\text { - Acesso à equipe de cuidados paliativos. } \\
\text { - Plano de cuidados individualizado e flexível. } \\
\text { - Minimização de tratamentos fúteis. }\end{array}$ \\
\hline $\begin{array}{l}\text { Medline, BMJ Support } \\
\text { Palliat Care, 2017, } \\
\text { Austrália }^{25}\end{array}$ & $\begin{array}{l}\text { Explorar o ponto de vista de médicos } \\
\text { de emergência sobre suas habilidades, } \\
\text { papel e experiência no cuidado de } \\
\text { pessoas com câncer avançado. }\end{array}$ & $\begin{array}{l}\text { - Controle dos sintomas. } \\
\text { - Gestão em rede de cuidados. }\end{array}$ \\
\hline $\begin{array}{l}\text { Medline, Am J Hosp } \\
\text { Palliat Care, 2017, } \\
\text { Inglaterra }{ }^{26}\end{array}$ & $\begin{array}{l}\text { Explorar as razões pelas quais } \\
\text { pacientes com necessidades de } \\
\text { cuidados paliativos buscam o } \\
\text { departamento de emergência. }\end{array}$ & $\begin{array}{l}\text { - Controle dos sintomas. } \\
\text { - Gestão em rede de cuidados. }\end{array}$ \\
\hline $\begin{array}{l}\text { Medline, Age Ageing, } \\
\text { 2017, Inglaterra, Irlanda } \\
\text { e EUA }^{27}\end{array}$ & $\begin{array}{l}\text { Identificar desafios e facilidades para o } \\
\text { empoderamento de pessoas idosas } \\
\text { com doença avançada e o impacto } \\
\text { dos cuidados paliativos. }\end{array}$ & $\begin{array}{l}\text { - Equipe especializada. } \\
\text { - Comunicação empática. } \\
\text { - Plano de cuidados individualizado e flexível. } \\
\text { - Acesso à equipe de cuidados paliativos. }\end{array}$ \\
\hline $\begin{array}{l}\text { Medline, Palliat Med, } \\
\text { 2018, Austrália }{ }^{28}\end{array}$ & $\begin{array}{l}\text { Explorar experiências e percepções } \\
\text { de pacientes com câncer avançado } \\
\text { e cuidadores que buscam o serviço } \\
\text { de emergência. }\end{array}$ & $\begin{array}{l}\text { - Controle dos sintomas. } \\
\text { - Comunicação empática. } \\
\text { - Gestão em rede de cuidados. } \\
\text { - Plano de cuidados individualizado e flexível. }\end{array}$ \\
\hline $\begin{array}{l}\text { Medline, Support Care } \\
\text { Cancer, 2018, Inglaterra }{ }^{29}\end{array}$ & $\begin{array}{l}\text { Explorar opiniões e experiências } \\
\text { de pacientes do departamento } \\
\text { de emergência posteriormente } \\
\text { internados. }\end{array}$ & $\begin{array}{l}\text { - Controle dos sintomas. } \\
\text { - Comunicação empática. } \\
\text { - Gestão em rede de cuidados. } \\
\text { - Plano de cuidados individualizado e flexível. }\end{array}$ \\
\hline
\end{tabular}

\section{Discussão}

A análise do modo como as equipes de emergência atendem pacientes em cuidados paliativos possibilitou responder à questão norteadora do estudo e identificar especificidades ou pontos de intersecção. Os aspectos mais evidentes foram: o cuidado pautado num plano individualizado e flexível, a gestão de rede de cuidados e o acesso a equipes de cuidados paliativos 3 ,6-8,10-15,17,20-21,24-29.

A necessidade de cuidado individualizado e flexível justifica-se pelo perfil dos pacientes, que muitas vezes chegam com diagnóstico de doença crônica e avançada, com histórico, em geral, de assistência incongruente, marcada por métodos invasivos, excesso de uso de tecnologias e sofrimento ignorado ou, pior, aumentado por práticas de distanásia ${ }^{14}$.

O encaminhamento precoce para equipe de interconsulta pode reduzir tanto o tempo de espera ${ }^{15}$ como o tempo de internação hospitalar. Um dos estudos identificou que um grupo de pacientes com acesso a equipe de interconsulta na emergência teve o tempo de hospitalização reduzido em 3,6 dias, em comparação com outro grupo que só passou por interconsulta após internação ${ }^{6}$.

A resistência em dar início à abordagem paliativa na emergência pode estar relacionada a atitudes e crenças dos profissionais sobre o processo de adoecimento, assim como a uma compreensão equivocada da emergência como setor de dinâmica acelerada, no qual não haveria tempo para a interação entre equipe, paciente e família. Assim, pacientes com doenças crônicas avançadas, como os portadores de demência e câncer, não são vistos como sujeitos que vivenciam um sofrimento ativo, causado por eventos agudos, e que precisam de estabilidade clínica e plano de cuidados individualizado e flexível para retornar ao seu quadro basal ${ }^{6,14}$. 
Vale destacar que esses pacientes buscam a emergência por diversos motivos: ansiedade com as crises agudas; falta de direcionamento prévio na rede de atenção primária; sentimento de segurança e familiaridade com o ambiente hospitalar; e, em muitas situações, dificuldade de acesso à atenção básica, especialmente em casos urgentes ou horários em que esses serviços não funcionam ${ }^{23}$.

Estudos apontam que as justificativas dos profissionais de emergência para não realizar a interconsulta se pautam em definições empíricas sobre a pertinência da conduta e na disponibilidade em iniciar o diálogo com a família 6,14,24. No entanto, cabe ressaltar que a participação da família e, principalmente, do paciente nas decisões assistenciais é um direito que deve ser assegurado pelos profissionais. Orientar e esclarecer as dúvidas de usuários e familiares é torná-los participantes do cuidado. Embora não decida questões técnicas, a família é coparticipe na tomada de decisão ${ }^{30}$.

Como abordagem ativa e multidisciplinar, os cuidados paliativos quebram paradigmas, defendendo um novo modelo centrado na autonomia do paciente, e não na equipe ou na doença, em prol da qualidade de vida e de resultados que visam o conforto e a dignidade humana ${ }^{22}$. Entretanto, pesquisa realizada em hospital australiano aponta que ainda há relutância em incluir esses cuidados na emergência, apesar das discussões já em marcha sobre a necessidade de revisar sistemas de modo a incluir a assistência paliativa ${ }^{25}$.

Os cuidados paliativos na emergência são marcados por dificuldades na comunicação, no reconhecimento da empatia como aspecto fundamental e na identificação de pacientes elegíveis $^{1-3,8-13,15-16,18-20,27-29}$. Embora reconheçam a necessidade dos cuidados paliativos na emergência, os profissionais não se sentem preparados para oferecê-los, em especial no que diz respeito a habilidades de comunicação, à capacidade de abordar questões relativas ao fim da vida e a conhecimentos para identificar e encaminhar para interconsulta os pacientes que precisam desses cuidados ${ }^{12}$.

Estudo realizado na Tailândia buscou descrever a experiência de enfermeiras no cuidado a pacientes críticos na emergência. Os resultados apontam para a necessidade de qualificar essas profissionais. As enfermeiras afirmam que o tempo em que o paciente permanece na emergência é muito curto, e por isso os cuidados paliativos não são priorizados. Sedação e medicamentos utilizados para aliviar a dor nunca são prescritos. Tais cuidados demandariam tempo e entrariam em conflito com a recomendação de transferir os pacientes o mais rápido possível para outras unidades, ou liberá-los para retornar a suas residências ${ }^{20}$.

No entanto, medidas práticas, com bons resultados, poderiam ser empreendidas, como fornecer um local reservado, com privacidade, para que paciente e família vivam integralmente o momento singular da morte, e reconhecer as diretivas antecipadas de vontade (manifestadas por testamento vital ou mandato duradouro) como instrumento que contempla a vontade do paciente em relação a tratamentos. Também seria importante, durante a formação dos profissionais de saúde, desenvolver habilidades de comunicação e formas de lidar emocionalmente com más notícias ${ }^{20-21}$.

Pesquisa com enfermeiras e médicos de emergência espanhóis que tiveram formação especializada aponta que esses profissionais se sentem mais confortáveis para lidar com pacientes com doenças terminais. Por outro lado, a falta de uma cultura de cuidados paliativos, conscientização, comunicação empática e treinamento profissional dificulta tal abordagem ${ }^{21}$.

É importante esclarecer que a comunicação sobre cuidados paliativos na emergência não pode seguir os mesmos critérios utilizados no modelo tradicional das unidades de internação ${ }^{3,7}$. Dentre as estratégias que também podem funcionar na emergência estão: distinguir cuidados paliativos de cuidados paliativos em fim de vida; proceder à abordagem inicial com uma equipe formada por médicos e enfermeiros; reconhecer as diretivas antecipadas de vontade; aperfeiçoar a formação da equipe; e ter um profissional que exerça liderança, sensibilizando os companheiros e mostrando como vencer barreiras ${ }^{5}$. Sempre que possível, o paciente e sua família também devem ser incluídos na tomada de decisão, assim como é preciso que capelães, psicólogos e assistentes sociais participem do cuidado ${ }^{22}$.

A última síntese aponta para o controle de sintomas. Esse ponto é importante, uma vez que pacientes descrevem suas experiências no atendimento como um momento de ansiedade e incerteza associadas à longa espera antes do manejo dos sintomas ${ }^{27}$. Os sintomas de ordem física mais relatados foram: dor, dispneia, náusea, vômito e constipação ${ }^{8,10,26}$. Contudo, há também questões de ordem 
emocional e social: ansiedade relacionada ao avanço da doença; busca recorrente pela emergência diante de crises agudas; sentimentos de segurança e familiaridade com o ambiente hospitalar; e dificuldades de acesso a serviços de atenção primária ${ }^{23}$.

Estudo realizado na Irlanda aponta que até $94 \%$ das pessoas que chegam à emergência com os sintomas supramencionados permanecem em observação por um tempo médio de nove horas. Todavia, 51,5\% desses pacientes não precisariam buscar a emergência se a gestão dos cuidados domiciliares fosse ativa e resolutiva ${ }^{8}$. Outro estudo, desenvolvido na Inglaterra, aponta que 83\% dos atendimentos exigem cuidados emergenciais diante de crise aguda, e que as necessidades das pessoas atendidas não são supridas pela atenção primária ou domiciliar. Assim, a emergência tem papel fundamental no gerenciamento de crises agudas e deve ser reconhecida como uma porta de entrada à rede de saúde ${ }^{26}$.

A excelência no atendimento passa por reconhecer as necessidades do paciente no momento de crise aguda, o que exige da equipe capacidade de comunicação empática e de identificar os objetivos inerentes ao atendimento e ao controle dos sintomas ${ }^{9}$. Nesse sentido, é preciso ter cautela com a recomendação de que o paciente busque a rede de atenção primária, ambulatorial ou domiciliar, haja vista que a agudização dos quadros crônicos é constante e nem sempre é resolvida em ambientes que não sejam a emergência. Assim, equivocam-se os profissionais que afirmam que pacientes em cuidados paliativos não precisam de unidades de emergência e devem ser atendidos apenas na rede de atenção primária ou ambulatorial ${ }^{26}$.

A competência dos profissionais no manejo de sintomas também tem sido apontada como ponto forte dos cuidados paliativos na emergência ${ }^{9,18,22,23,25}$. Um estudo apontou que $84,2 \%$ dos médicos se sentiam confortáveis em cuidar de pacientes em estágio avançado da doença ${ }^{13}$. Em outra pesquisa, $64,8 \%$ dos enfermeiros consideraram recompensador 0 atendimento a esse perfil de paciente. $O$ enfermeiro tem sido apontado como elo da equipe na prestação dos cuidados, interligando-se com o paciente e sua família, otimizando os cuidados de conforto e garantindo um ambiente digno e humano ${ }^{22}$.

\section{Considerações finais}

A pesquisa evidenciou que os serviços de emergência costumam ser vistos como espaços apenas para ações rápidas, o que traz obstáculos para o conforto do paciente e a preservação da dignidade, justamente em um espaço teoricamente projetado para salvar vidas. Assim, muitos profissionais ainda não reconhecem a emergência como local onde é possível oferecer cuidados paliativos. Como principais medidas que poderiam reverter esse cenário, vale destacar: plano de cuidados individualizado e flexível, gestão de redes de cuidado, acesso à equipe de cuidados paliativos, comunicação empática, identificação de pacientes elegíveis e controle de sintomas.

Muitos pacientes e familiares se dirigem à emergência buscando segurança. Assim, é fundamental que a equipe de emergência reconheça a importância dos cuidados paliativos, redirecionando o cuidado concentrado em "salvar vidas" para um cuidado que preserve a dignidade humana, reconhecendo a morte como parte do ciclo da vida.

Entre as limitações do presente estudo, destacam-se a utilização de apenas cinco bases de dados, não incluindo a Web of Science e a Embase, e a ausência de produções nacionais. No entanto, apesar dessas limitações, espera-se que o artigo seja uma oportunidade de reflexão sobretudo para os profissionais da emergência, instigando-os a conhecer os princípios filosóficos dos cuidados paliativos. O objetivo é que esses princípios pautem sua atuação mesmo em uma dinâmica acelerada como a das emergências, proporcionando a pacientes e familiares cuidados de fim de vida resolutivos, com compaixão, conforto e dignidade.

\section{Referências}

1. Mierendorf SM, Gidvani V. Palliative care in the emergency department. Perm J [Internet]. 2014 [acesso 10 jan 2019];18(2):77-85. DOI: 10.7812/TPP/13-103 
2. Bailey C, Murphy R, Porock D. Trajectories of end-of-life care in the emergency department. Ann Emerg Med [Internet]. 2011 [acesso 10 jan 2019];57(4):362-9. DOI: 10.1016/j.annemergmed.2010.10.010

3. Lamba S, Nagurka R, Zielinski A, Scott SR. Palliative care provision in the emergency department: barriers reported by emergency physicians. J Palliat Med [Internet]. 2013 [acesso 10 jan 2019];16(2):143-7. DOI: 10.1089/jpm.2012.0402

4. Hopia H, Latvala E, Liimatainen L. Reviewing the methodology of an integrative review. Scand J Caring Sci [Internet]. 2016 [acesso 10 jan 2019];30(4):662-9. DOI: 10.1111/scs.12327

5. Rosenberg M, Rosenberg L. Integrated model of palliative care in the emergency department. West J Emerg Med [Internet]. 2013 [acesso 10 jan 2019];14(6):633-6. DOI: 10.5811/westjem.2013.5.14674

6. Wu FM, Newman JM, Lasher A, Brody AA. Effects of initiating palliative care consultation in the emergency department on inpatient length of stay. J Palliat Med [Internet]. 2013 [acesso 10 jan 2019];16(11):1362-7. DOI: 10.1089/jpm.2012.0352

7. Grudzen CR, Richardson LD, Major-Monfried H, Kandarian B, Ortiz JM, Morrison RS. Hospital administrators' views on barriers and opportunities to delivering palliative care in the emergency department. Ann Emerg Med [Internet]. 2013 [acesso 10 jan 2019];61(6):654-60. DOI: 10.1016/j.annemergmed.2012.06.008

8. Wallace EM, Cooney MC, Walsh J, Conroy M, Twomey F. Why do palliative care patients present to the emergency department? Avoidable or unavoidable? Am J Hosp Palliat Care [Internet]. 2013 [acesso 10 jan 2019];30(3):253-6. DOI: 10.1177/1049909112447285

9. Bailey FA, Williams BR, Woodby LL, Goode PS, Redden DT, Houston TK et al. Intervention to improve care at life's end in inpatient settings: the BEACON trial. J Gen Intern Med [Internet]. 2014 [acesso 10 jan 2019];29(6):836-43. DOI: 10.1007/s11606-013-2724-6

10. Yildirim B, Tanriverdi O. Evaluation of cancer patients admitted to the emergency department within one month before death in Turkey: what are the problems needing attention? Asian Pac J Cancer Prev [Internet]. 2014 [acesso 10 jan 2019];15(1):349-53. DOI: 10.7314/apjcp.2014.15.1.349

11. Lane H, Weil J, Jelinek GA, Boughey M, Marck CH, Weiland TJ et al. Ideal care and the realities of practice: interdisciplinary relationships in the management of advanced cancer patients in Australian emergency departments. Support Care Cancer [Internet]. 2014 [acesso 10 jan 2019];22(4):1029-35. Disponível: https://bit.ly/3tnLfJ6

12. Shearer FM, Rogers IR, Monterosso L, Ross-Adjie G, Rogers JR. Understanding emergency department staff needs and perceptions in the provision of palliative care. Emerg Med Australas [Internet]. 2014 [acesso 10 jan 2019];26(3):249-55. DOI: 10.1111/1742-6723.12215

13. Marck CH, Weil J, Lane H, Weiland TJ, Philip J, Boughey M, Jelinek GA. Care of the dying cancer patient in the emergency department: findings from a National survey of Australian emergency department clinicians. Intern Med [Internet]. 2014 [acesso 10 jan 2019];44(4):362-8. DOI: 10.1111/imj.12379

14. Ouchi K, Wu M, Medairos R, Grudzen CR, Balsells H, Marcus D et al. Initiating palliative care consults for advanced dementia patients in the emergency department. J Palliat Med [Internet]. 2014 [acesso 10 jan 2019];17(3):346-50. DOI: 10.1089/jpm.2013.0285

15. Kistler EA, Sean MR, Richardson LD, Ortiz JM, Grudzen CR. Emergency department-triggered palliative care in advanced cancer: proof of concept. Acad Emerg Med [Internet]. 2015 [acesso 10 jan 2019];22(2):237-9. DOI: 10.1111/acem.12573

16. Weil J, Weiland TJ, Lane H, Jelinek GA, Boughey M, Marck $\mathrm{CH}$ et al. What's in a name? A qualitative exploration of what is understood by "palliative care" in the emergency department. Palliat Med [Internet]. 2015 [acesso 10 jan 2019];29(4):293-301. DOI: 10.1177/0269216314560801

17. Weiland TJ, Lane H, Jelinek GA, Marck CH, Weil J, Boughey M, Philip J. Managing the advanced cancer patient in the Australian emergency department environment: findings from a national survey of emergency department clinicians. Int J Intern Emerg Med [Internet]. 2015 [acesso 10 jan 2019];8. DOI: 10.1186/s12245-015-0061-8

18. Russ A, Mountain D, Rogers IR, Shearer F, Monterosso L, Ross-Adjie G, Rogers JR. Staff perceptions of palliative care in a public Australian, metropolitan emergency department. Emerg Med Australas [Internet]. 2015 [acesso 10 jan 2019];27(4):287-94. DOI: 10.1111/1742-6723.12428 
19. Fassier T, Valour E, Colin C, Danet F. Who am I to decide whether this person is to die today? Physicians' life-or-death decisions for elderly critically ill patients at the emergency department-ICU interface: a qualitative study. Ann Emerg Med. [Internet]. 2016 [acesso 10 jan 2019];68(1):28-39. DOI: 10.1016/ j.annemergmed.2015.09.030

20. Kongsuwan W, Matchim Y, Nilmanat K, Locsin RC, Tanioka T et al. Lived experience of caring for dying patients in emergency room. Int Nurs Rev [Internet]. 2016 [acesso 10 jan 2019]; 63(1): 132-8. DOI: 10.1111/inr.12234

21. Granero-Molina J, Díaz-Cortés MDM, Hernández-Padilla JM, García-Caro MP, Fernández-Sola C. Loss of dignity in end-of-life care in the emergency department: a phenomenological study with health professionals. J Emerg Nurs [Internet]. 2016 [acesso 10 jan 2019];42(3):233-9. DOI: 10.1016/ j.jen.2015.10.020

22. Rojas E, Schultz R, Linsalata HH, Sumberg D, Christensen M, Robinson C, Rosenberg M. Implementation of a life-sustaining management and alternative protocol for actively dying patients in the emergency department. J Emerg Nurs [Internet]. 2016 [acesso 10 jan 2019];42(3):201-6. DOI: 10.1016/ j.jen.2015.11.006

23. Henson LA, Higginson IJ, Daveson BA, Ellis-Smith C, Koffman J, Morgan M, Gao W. 'I'Il be in a safe place': a qualitative study of the decisions taken by people with advanced cancer to seek emergency department care. BMJ Open [Internet]. 2016 [acesso 10 jan 2019];6(11). DOI: 10.1136/bmjopen-2016-012134

24. Kim, Kenneth; Chakravarthy, Bharath; Anderson, Craig; Liao, Solomon. To intubate or not to intubate: emergency medicine physicians' perspective on intubating critically III, terminal cancer patients. J Pain Symptom Manage [Internet]. 2017 [acesso 10 jan 2019];54(5):654-60. DOI: 10.1016/ j.jpainsymman.2017.07.038

25. Jelinek GA, Marck CH, Weil J, Lane H, Philip J, Boughey M, Weiland TJ. Skills, expertise and role of Australian emergency clinicians in caring for people with advanced cancer. BMJ Support Palliat Care [Internet]. 2017 [acesso 10 jan 2019];7(1):81-7. DOI: 10.1016/j.jpainsymman

26. Green E, Ward S, Brierley W, Riley B, Sattar H, Harris T. "They shouldn't be coming to the ED, should they?": a descriptive service evaluation of why patients with palliative care needs present to the emergency department. Am J Hosp Palliat Care [Internet]. 2017 [acesso 10 jan 2019];34(10):984-90. DOI: $10.1177 / 1049909116676774$

27. Selman LE, Daveson BA, Smith M, Johnston B, Ryan K, Morrison RS et al. How empowering is hospital care for older people with advanced disease? Barriers and facilitators from a cross-national ethnography in England, Ireland and the USA. Age Ageing [Internet]. 2017 [acesso 10 jan 2019];46(2):300-9. DOI: 10.1093/ageing/afw193

28. Philip J, Remedios C, Breen S, Weiland T, Willenberg L, Boughey $M$ et al. The experiences of patients with advanced cancer and caregivers presenting to emergency departments: a qualitative study. Palliat Med [Internet]. 2018 [acesso 10 jan 2019];32(2):439-46. DOI: 10.1177/0269216317735724

29. Chen H, Johnson M, Boland E, Seymour J, Macleod U. Emergency admissions and subsequent inpatient care through an emergency oncology service at a tertiary cancer centre: service users' experiences and views. Support Care Cancer [Internet]. 2018 [acesso 10 jan 2019];27:451-60. DOI: 10.1007/s00520-018-4328-5

30. Cogo SB, Lunardi VL, Quintana AM, Girardon-Perlini NMO, Silveira RS. Assistência ao doente terminal: vantagens na aplicabilidade das diretivas antecipadas de vontade no contexto hospitalar. Rev Gaúcha Enferm [Internet]. 2017 [acesso 10 jan 2019];38(4). DOI: 10.1590/1983-1447.2017.04.65617 
Maria Olivia Sobral Fraga de Medeiros - Mestre - poppmedeiros@yahoo.com.br

(D) 0000-0001-6811-378X

Mariana do Valle Meira - Mestranda - mariana.meira12@gmail.com

(D) 0000-0003-0280-9455

Jacilene Santiago do Nascimento Trindade do Santos - Mestre - jacilenesnts@hotmail.com

(D) 0000-0001-9535-5565

Larissa Chaves Pedreira - Doutora - Ichavesp@ufba.br

(D) 0000-0001-8939-324X

Anelise Coelho da Fonseca - Doutora - anelise1976@gmail.com

(D) 0000-0001-6027-2778

Rudval Souza da Silva - Doutor - rudsouza@uneb.br

(D) 0000-0002-7991-8804

Correspondência

Rudval Souza da Silva - Universidade do Estado da Bahia. Colegiado de Enfermagem. Campus VII.

Rodovia Lomanto Júnior, BR 407, km 127 CEP 48970-000. Senhor do Bonfim/BA, Brasil.

Participação dos autores

Maria Olivia Sobral Fraga de Medeiros, Mariana do Valle Meira e Rudval Souza da Silva redigiram o manuscrito. Jacilene Santiago do Nascimento Trindade dos Santos, Larissa Chaves Pedreira e Anelise Coelho da Fonseca contribuíram com a revisão final do texto. 